Fibromyalgia in people with more than 65 years, is in our group very low. This result can be real or because from the primary care this pathology in this group of people is not considered so important as others.

\section{OP0079 PAIN, PSYCHOLOGICAL STATUS, SLEEP DISTURBANCE, AND SERUM CONCENTRATION OF 5- HYDROXYTRYPTAMINE IN PATIENTS WITH PRIMARY FIBROMYALGIA AND CHRONIC LOW BACK PAIN - A COMPARATIVE STUDY}

${ }^{1} \mathrm{PZ}$ Hrycaj, ${ }^{2} \mathrm{~B}$ Hauns, ${ }^{2} \mathrm{~K}$ Ovenhausen, ${ }^{2} \mathrm{P}$ Mennet, ${ }^{2} \mathrm{~W}$ Müller. ${ }^{1}$ Department of Rheumatology and Clinical Immunology, Karol Marcinkowski University School of Medical Sciences, Poznan, Poland; ${ }^{2}$ Department Für Die Grundlagenforschung in Der Rheumatologie, Hochrhein-Institut Für Rehabilitationsforschung E. V., Bad Säckingen, Germany

\subsection{6/annrheumdis-2001.587}

Background Several reports suggest that, in most cases, fibromyalgia syndrome (FS) begins as a localised pain syndrome which may precede for months or years the development of the full-blown disease. Thus, some patients who currently present with chronic low back pain (LBP) may in future develop FS.

Objectives To study FS signs and symptoms and serum serotonin (5-hydroxytryptamine, 5-HT) in patients with chronic low back pain syndrome (LBP) in order to identify possible predictors of FS development.

Methods Comparative and correlation analysis of clinical data (pain intensity, tender point count, average pain threshold, functional symptoms, levels of depression and anxiety, feeling of well-being, measures of sleep disturbance) and serum 5-HT in patients with FS $(\mathrm{n}=34)$ and LBP $(\mathrm{n}=46)$.

Results FS patients had higher intensity of pain, higher tender point count, lower average pain threshold, more functional symptoms, higher scores on depression and anxiety scales, decreased feeling of well-being, and more sleep troubles than those with LBP ( $p<0.0001$ for all comparisons). In both groups, there was a strong interrelationship between functional symptoms, depression, anxiety, feeling of well-being, and the sleep disturbance. Serum 5-HT was lower in FS group when compared to healthy sex- and age-matched controls $(n=26, p$ $<0.03)$. Although low serum 5 -HT was found in some patients with LBP, the median serum level of the monoamine did not differ significantly from that found in FS and controls. There was no relationship between serum 5-HT and the clinical characteristics in the LBP group. Women with LBP had higher count of tender points $(\mathrm{p}<0.0001)$, lower pain threshold $(\mathrm{p}<0.0001)$, higher median level of depression $(p<0.005)$, and more disturbed sleep $(\mathrm{p}<0.05)$ than men.

Conclusion As expected, striking differences in clinical characteristics were found between FS and LBP with greater disturbance seen with the FS group. Our study confirms earlier reports suggesting lowered serum 5-HT in FS and provide no support to the hypothesis that there may be a general 5 -HT deficiency in LBP. Higher tender point count, decreased pain threshold, depression, and sleep disturbance are possible predictors of FS development in LBP and should be investigated in further prospective research.

\section{OP0081 THE EFFECTIVENESS OF AEROBIC EXERCISE AND EDUCATION IN FIBROMYALGIA SYNDROME}

O Kuru, N Yildiz, A Bilgici. Physical Medicine and Rehabilitation, Ondokuz Mayis University Medical Faculty, Samsun, Turkey

\subsection{6/annrheumdis-2001.588}

Background Fibromyalgia is a common syndrome found predominantly in women. It is characterised by chronic, diffuse, musculoskeletal pain and a sleep disorder, without evidence of arthritis or myositis.

Objectives The purpose of this study was to compare the effectiveness of aerobic exercise and education with the control group in fibromyalgia syndrome and to evaluate the patients treated with this method after the treatment program.

Methods Sixty women, aged 18-55 years, diagnosed as fibromyalgia according to 1990 ACR criteria were randomly divided into two groups. Group I was given aerobic exercise and stretching exercise for 8 weeks following an education program. Group II was the control group. Both groups took sertralin $50 \mathrm{mg} /$ day. The patients were evaluated before and after the treatment. We used VAS, number of tender points, total myalgic score for pain, Beck depression inventory (BDI) for depression, Patient and doctor global assessment scale, Fibromyalgia attitudes index (FAI) for global assessment, Fibromyalgia impact questionnaire (FIQ), Health assessment questionnaire (HAQ) for functional evaluation, Astrand test for aerobic capacity.

Results The results of VAS, number of tender points, total myalgic score, BDI, FAI, FIQ, HAQ and patient and doctor global assessment scale showed significant improvement in both groups $(\mathrm{p}<0.001, \mathrm{p}<0.01)$. In the first group, the results of VO2max showed significant improvement $(\mathrm{p}<0.001)$. The results of some groups of FIQ, SELF function subscale, FAI and BDI were not statistically different among the two groups $(p>0.05)$. We found significant improvement in other parameters in exercise group than the control group.

Conclusion Fibromyalgia is a multifactorial syndrome, and the best treatment will encompass multiple strategies, that include education, stress management treatment and aerobic exercise.

\section{OP0082 CENTRAL SENSITISATION AND CLINICAL PAIN SEVERITY IN CYP2D6 PHENOTYPED FIBROMYALGIA (FM) PATIENTS}

${ }^{1} \mathrm{JA}$ Desmeules, ${ }^{2} \mathrm{C}$ Cedraschi, ${ }^{2} \mathrm{E}$ Baumgartner, ${ }^{2} \mathrm{~A}$ Finckh, ${ }^{3} \mathrm{P}$ Cohen, ${ }^{1} \mathrm{P}$ Dayer, ${ }^{2} \mathrm{TL}$ Vischer. ${ }^{1}$ Clinical Pharmacology and Toxicology; ${ }^{2}$ Rheumatology; ${ }^{3}$ Reeducation Clinic, Geneva University Hospitals, Geneva, Switzerland

\subsection{6/annrheumdis-2001.589}

Background Nociceptive processing in fibromyalgia patients could be altered by aberrant central mechanisms.

Objectives R-III nociceptive reflex can be used to assess nociceptive processing and to provide indirect evidence of central pain sensitisation.

Methods 95 of 150 FM included in an ongoing RCT of an exercise program vs 40 matched controls (C) were evaluated. Pain severity was assessed by number of tender points (TP), myalgic score (MS) and physician global impression (PGI). Patient's ratings included a VAS and the regional pain score (RPS). FM and $\mathrm{C}$ were assessed by central (R-III) and peripheral (thermotests) quantitative sensory testing (QST). QST and clinical parameters were correlated to CYP2D6 phenotypes. 